\title{
CHALLENGES
}

\section{Challenges for child health in Australia}

\author{
R Kim Oates
}

In any current discussion of child health in Australia, the financial aspect is usually predominant. Governments tell us that there has been a 'blow-out' in health costs. This argument is not supported by the facts which show that health expenditure in Australia has merely kept up with inflation, remaining at between $7 \cdot 5 \%$ and $8 \cdot 1 \%$ of gross domestic product for the last 15 years. The blow-out in health costs is in fact similar to the blow-out in the cost of ball point pens and breakfast cereal. For child health the challenge is how to get an adequate share of the health budget and how to use it most appropriately.

Of course there are many other clinical challenges which confront Australian paediatricians. Relatively little impact has been made on the two biggest causes of death of children after the first month of life, the suddent infant death syndrome in the first year and accidents beyond the first year. Even up to 1 month of age, while there has been considerable progress in the intensive care of extremely low birthweight infants, there has been far less progress in preventing the premature births which lead to these problems. Other major health challenges for Australian paediatricians, in terms of size and seriousness, include better understanding and improved management of asthma, a reduction in the number of cases of child abuse and neglect, and providing care for the increasing number of children surviving, often into adult life, with chronic handicaps.

While none of these challenges have simple answers, the four headings in this paper: health costs; hospitals, their communities and health delivery; child health education; and a national child health policy, are relevant to progress in these areas.

\section{Health costs}

The cost of health in Australia is borne largely by the tax payer. Taxation funds Medicare (a scheme of universal health insurance which covers the costs of hospital care in the nonprivate sector and $85 \%$ of the 'common fee' for medical consultations and surgical procedures), the Pharmaceutical Benefits Scheme, and some government funded research. The federal government also funds the bulk of hospital running costs through grants to the states although the states determine the allocation of these funds to hospitals.

Assuming that there will be no increase in the health budget, the problem for child health is to measure the effectiveness of what we do so that health services for children are not dis- advantaged when health resources are allocated. While it is true that children are our future and that promotion of good child health is likely to reduce future adult health costs, facts are needed to support this argument. For children's health services to mount an effective argument for an adequate share of the health budget, effective outcome measures must be developed. Effective outcome measures enable logical decisions to be made about which areas of child health care may be able to be given up if they are found to be of little value and which areas need to be enhanced and improved, while at the same time providing a way of establishing the effectiveness of the newly introduced technology.

In an attempt to measure the activity of hospitals, and to determine their funding, Australia is looking at the introduction of diagnostic related groups (DRGs) for hospitals. As DRGs use average length of stay as a measure of cost and as children's hospitals have long had emphasis on children being in hospital for the minimal time, there is a danger that DRGs may disadvantage children's hospitals. Because adults in hospital often have multiple problems, while most children usually have only one or two problems, the DRG loading given to children's conditions for complexity is lower. Further, the range and complexity of some paediatric problems was not allowed for in the initial DRG formulation, although a revised version is more acceptable. The Australian Association of Paediatric Teaching Centres is currently assessing how DRGs can be used so as not to disadvantage children's hospitals.

Another way of looking at outcome is to use quality adjusted life years (QALYS). Quality of adjusted life looks at physical functioning, the person's role in society, their relationship to society and their health, with these measures being adjusted for longevity. This allows a comparison to be made between outcome and expenditure for different diseases. It can be a powerful tool in arguing for increased paediatric funding as most children who survive medical and surgical problems do so with a good quality of life and long life expectancy in comparison with the results of treating many adult diseases. QALYS can also can be used to make value for money comparisons between different paediatric treatments such as comparing costs and value of school screening procedures, or even neonatal intensive care, with the cost and benefit of introducing Haemophilus influenzae immunisation.

The development of efficient outcome measures will improve the quality of care we 
provide. Health costs are partly determined by the fact that health providers, particularly hospitals, have done things in the same way for such a long time that it is comfortable to keep things the way they are. A squeeze on the health budget has certainly raised the discomfort level and has been necessary to encourage innovative, lateral thinking as to the most efficient ways of providing health care for children.

While a proportion of the health budget should be set aside for research into outcome measures, laboratory and clinical research is largely funded by the federal government through the National Health and Medical Research Council's peer reviewed, competitive grant process. Federal funds for health research have been increased by $8 \%$ per annum in real terms since 1989 with initiative in such areas as asthma, the health needs of disadvantaged groups, public health research and opportunities for training young researchers. However, research expenditure is only $1.5 \%$ of total health expenditure, whereas the internationally recommended level is $2 \%$. For child health, the challenge is for paediatric researchers to compete effectively with the other, often more research oriented medical disciplines, while a problem for all researchers is that research salaries are becoming even less competitive in the market place.

\section{Hospitals, their communities, and health delivery}

There is sometimes talk about hospital paediatrics and community paediatrics as though they were two separate entities. Children's hospitals and children's units in general hospitals are community resources, just as schools and shopping centres are community resources. It would be advantageous for the distinction between hospital and community paediatrics to merge, with hospital and community services sharing some staff and resources, while recognising that certain conditions can obviously only be managed in hospitals while for other problems, children are best served by having their health delivery in the community. Some of the challenges that children's hospitals face within their own walls are those of chronic illness causing an increased demand on resources. This includes children with long term handicaps who may require frequent hospital admissions, although occasionally these admissions are for respite and could be better managed in a more homely, respite atmosphere than in a high technology teaching hospital. Children's hospitals are increasingly looking to the community to have some of the needs of these chronically handicapped children met, such as the small, but increasing number of children being ventilated at home. An example is a quadriplegic child with no spontaneous respiratory function who, having had his immediate care in hospital, is able to be cared for at home, with home visiting from a hospital or community based service with appropriate expertise.

Many children with long term chronic illness are now surviving beyond the paediatric age group, so that subsequent hospital and com- munity care will be in adult hospitals or from adult based community services. These include older teenagers with cystic fibrosis and spina bifida, conditions which may be poorly understood by adult specialists and where access to the comprehensive paramedical services and team approach of a children's hospital may not be part of the philosophy of the adult centre. The transition of our patients to adult care is rather like the problem parents face as their children grow up. As our patients grow older we should be teaching them to be less dependent on paediatric services and be willing to start letting go while ensuring that we communicate with and educate our adult colleagues in the hospital and the community, so that our patients can move on to a level of care sufficient for their needs. Regular meetings between paediatricians and adult physicians and transitional, joint clinics will help.

Children's hospitals in Australia have concentrated in the last 15-20 years on building up specialty units so that most have units representing all of the paediatric subspecialties. This has sometimes been at the expense of generalist services. The question is whether there is still room for general paediatricians in children's hospitals. The experience of adult teaching hospitals suggests there is. Most adult teaching hospitals no longer have general physicians so that patients, who may have multiple problems are admitted under the care of a subspecialist who is often unable to take an overview of the patient's problems and coordinate care. The general paediatrician in the teaching hospital is also necessary for role modelling. Most current paediatric trainees are likely to move into general or community paediatric areas as most of the subspecialty units are adequately filled by relatively young subspecialists.

While teaching hospitals have a major inpatient focus, a significant proportion of their work involves outpatients. One way of integrating hospital and community child health services is to decrease the length of stay even further with more emphasis on outreach services, utilising paediatric nursing and medical home care services combined with the development of protocols for high quality ambulatory care. This would achieve improved integration of hospital and community services with specialty staff, who may have an appointment in the community as well as in the hospital, being responsible for ensuring continuity of care using resources which move between the hospital and the community.

The challenges for community child health are even greater. The major one is to determine the effectiveness of community child health services. While screening surveillance is traditionally part of community child health, adequate information systems which give data about outcomes, rather than about throughputs have to be developed. There is little point in screening if we cannot measure the outcome of the screening process.

While it is difficult to measure the value of community child health services, particularly primary and secondary prevention and health promotion, ways of rigorously evaluating these 
services have to be found. Traditionally, community child health services have not attracted people with experience in investigation and evaluation. However, the newer generation of paediatricians includes some with expertise in epidemiology, research, and biostatistics. As they move into community child health, this will be one of their tasks. The initiative has been taken by the Australian College of Paediatrics by establishing a Faculty of Community Child Health to better integrate hospital and community services. This is particularly important in view of the opinion in some sections of government that doctors are not particulary necessary in child health and that ancillary medical staff, who may be cheaper to employ, can do many of their duties. While this view may have some validity, the generic health worker may have a little knowledge in a wide range of areas but often not enough to make real impact. The challenge for medical staff in community child health is to ensure that they develop methods of rigorous evaluation, quality assurance, and peer review to improve the status and quality of community based child health.

The challenge for hospital and community based child health services is improved integration. While the high and rigorous standards of paediatric teaching centres should move into the community, there is room for some community child health 'in-reach' into the teaching centres as the practical, feet on the ground approach of community based doctors to the problems of children and their families living in the real world has much to teach those working in hospitals.

\section{Child health education}

In Australia the primary care of children is provided by general practitioners. Most medical schools in Australia provide between nine and 11 weeks of paediatric teaching and experience. Unless graduates obtain further paediatric training they are likely to be poorly equipped to cope with the range of children's health problems. On the other hand, the steadily increasing number of paediatricians means that there is the potential for some paediatricians to set up in primary care practice rather than as consultants.

As teaching hospitals are the repository for much of the paediatric expertise, the question has to be asked as to why teaching hospitals still see children sent in with problems such as undiagnosed meningitis and poorly treated asthma. To be critical of the standard of child health care in general practice when it is the responsiblity of those with the expertise to ensure that standards of continuing education are high and widely avaliable in a realistic and attractive way, smacks of hypocrisy. However, the provision of continuing education is difficult in times of financial stringency when hospitals are struggling just to provide an adequate level of patient care. It also raises the problem of training versus service. What is the obligation of children's hospitals to provide teaching and training to those working in the community and to their house staff when house staff have to cope with a busy load and when health administrators may feel that house staff are employed primarily to look after patients? Even though there are many programmes of formal and informal teaching, there is a discrepancy between the conditions emphasised (by the nature of the case load) in teaching hospital training and the sort of child health problems seen by a general practitioner or paediatrician working in the community or a country centre. While the general practitioner needs to be familiar with common paediatric conditions and to know which childhood problems need expert help, and while the paediatrician will need to be familiar with the full range of paediatric conditions, may need to be able to initiate ventilation of neonates and cope with paediatric emergencies, there will be considerable emphasis in the community on behavioural and developmental problems in childhood, something which the acute teaching hospital situation gives little opportunity for mastering.

Although the general practitioner is the person best placed to provide primary paediatric care and while those with expertise in paediatrics have a responsibility to ensure that the general practioner's level of training is adequate, the large number of paediatricians currently being trained means that consultant positions will not always be available, so that some may be tempted to move into primary paediatric care. The Workforce Committee of the Australian College of Paediatrics has shown that while there are approximately 600 paediatricians in Australia there are approximately 190 paediatric registrars in training, representing a potential increase of $30 \%$ in the total number of paediatricians. Workforce projections, taking into account the age of consultant paediatricians in Australia, show that we are currently training almost double the number of paediatricians required. One partial solution is to reduce the number of paediatric training positions and to increase the expertise of general practitioners, by increasing the number of house staff positions for those aiming for a career in general practice. However, the problem is compounded by neonatal intensive care service requirements where paediatric registrars are appointed to meet these service needs (a paediatrician in training could spend two of the six years training in neonatal intensive care) so that in recent years, the number of registrar positions has increased, with many registrars then going on to complete paediatric training. The challenge is to look at ways of having some aspects of neonatal intensive care performed by others, perhaps suitably trained nurses.

\section{A national child health policy}

Australia does not have a national child health policy. The challenge to develop one is a current task of the Child Health Committee of the National Health and Medical Research Council and the Australian Association of Paediatric Teaching Centres. As well as having value in clarifying thinking and coordinating efforts, the development of a national child health policy for children has considerable political value as, 
once the major child health groups in the country agree on such a policy, political candidates and parties can be publicly asked how much they will support it.

Preliminary work on a national child health policy has outlined these goals.

(1) To develop access to adequate health services for all Australian children, regardless of their financial status or ethnic background, and in doing so, to identify disparity in health status between different community groups and work towards its correction. This is particulary relevant to the health of Aboriginal children where the perinatal and infant mortality rates are much higher than for non-Aboriginal children.

(2) To reduce the frequency of preventable premature mortality (from accidents and suicide), to establish national child safety standards, and support a national poisons information centre.

(3) To reduce the impact of disability. This includes a reduction in the occurrence of new disability by preventative measures as well as a reduction of the impact of established disability by early recognition along with appropriate interventions that are maintained and evaluated. Such conditions include congenital abnormalities, prematurity, chronic illness, intellectual and physical disability as well as learning disorders.

(4) To reduce the impact of vaccine preventable disease.

(5) To reduce the impact of diseases that occur in adulthood but which have their origins or early manifestations in childhood and adolescence. These include conditions associated with nutritional problems, diseases associated with alcohol and tobacco use, conditions associated with unprotected sexual activity, and those associated with poor cardiovascular fitness.

(6) To enhance family and social functioning including adequate parenting, the health needs of foster children and children of divorce, the many different types of families and cultures in
Australian society, as well as health problems associated with poor housing, low income, unemployment, and social and educational disadvantage.

These goals are a reminder that optimum child health is much broader than disease treatment and prevention. It means that as well as ensuring access to adequate standards of acute health care for all children, they should be adequately immunised, educated about the hazards of smoking, protected from abuse and neglect, have their learning difficulties addressed, and be protected from injury. Many of these priorities are clearly related to education and welfare, although they are an intregral part of promoting child health.

A national child health strategy must also look at Australia's relationship to Asia. For many years Asia was a place where Australian paediatricians travelling to Europe stopped while their plane was being refuelled. We are now developing a greater understanding of health care in the Asian region and realising that, as residents of this region, we should become more involved in training and education and in sharing knowledge and expertise.

It is difficult to make predictions about challenges for child health in Australia far into the future. When looking back over the last 30 years it becomes apparent that it would have been impossible to predict the amazing advances such as in diagnostic imaging and in molecular biology which have occurred throughout the world and in which Australia has shared. The only thing we can be sure about for the next 30 years is that once again it will probably be impossible to predict the extent of the changes which will occur in paediatrics. However the need for accountability, rigorous evaluation, equity and access of health care to all children will remain whatever the technological advances.

Many of the ideas in this paper are the results of helpfu discussions held with Drs W Butt, D Jolly, B Neal, F Oberklaid, and J Yu and Professors A Carmichael, L Landau, J Pearn, $P$ Phelan, D Roberton, and G Vimpani. 